\title{
Las juventudes en la historia Colombiana del siglo XIX y $\mathrm{XX}$
}

\section{The youths in the Colombian history of the 19th and 20th century}

\author{
Carlos Arturo Reina Rodríguez \\ Universidad Distrital "Francisco José de Caldas” (Bogotá, Colombia) \\ profesorcarlosreina@gmail.com \\ careina@udistrital.edu.co
}

\begin{abstract}
Resumen
Los estudios sobre la historia de la juventud en la historia colombiana son escasos. Existe un aparente desinterés por revisar el pasado de los sectores jóvenes, que contrasta con los abundantes estudios que desde la sociología y la antropología y en general las Ciencias Sociales, han hecho sobre las juventudes contemporáneas, que vinculan temas como las manifestaciones culturales urbanas, violencia y delincuencia juvenil, desempleo y migración. Este ensayo, tiene como primer objetivo, mostrar la presencia efectiva de los jóvenes en la historia colombiana. En segundo lugar, evidenciar su papel protagónico en la vida social, cultural y política del país, a través de una línea cronológica que divide el texto entre algunos referentes en el siglo XIX y otros que se han tomado en el siglo XX. Se trata de mostrar que, en el caso colombiano, como posiblemente en otros en la región, la presencia de los jóvenes ha sido importante y que es posible construir una historia cultural y social a su alrededor.
\end{abstract}

\section{Palabras clave}

Historia; Juventud; Colombia.

\begin{abstract}
Studies on the history of youth in Colombian history are scarce. There is an apparent lack of interest in reviewing the past of young sectors, which contrasts with the abundant studies that from sociology and anthropology and in general the Social Sciences have done on contemporary youth, which link issues such as urban cultural manifestations, violence and juvenile delinquency, unemployment and migration. This essay has as its first objective, to show the effective presence of young people in Colombian history. Secondly, to demonstrate its leading role in the social, cultural and political life of the country, through a chronological line that divides the text between some referents in the 19th century and others that have been taken in the 20th century. It is to show that, in the case of Colombia, as possibly in others in the region, the presence of young people has been important and that it is possible to build a cultural and social history around them.
\end{abstract}

\section{Keywords}

History; Youth; Colombia.

Esta obra está sujeta a la Licencia Reconocimiento-NoComercial-CompartirIgual 4.0 Internacional de Creative Commons. http://creativecommons.org/licenses/by-nc-sa/4.0/ 


\section{Las juventudes en la historia Colombiana del siglo XIX y XX}

\section{Presentación}

Podemos hallar rastros sobre los jóvenes en los estudios que tienen que ver con aspectos como familia, y población. Sabemos que estos aportan elementos importantes a la configuración de una historia de los jóvenes, pero la sola mención no basta para constituirse en una "historia", pues no constituyen su objeto de investigación. A esto se suma la dificultad para ubicar a los jóvenes, sus denominaciones en el tiempo y la forma como hicieron presencia en la historia del país. Algunas huellas se encuentran en biografías y autobiografías donde la juventud es señalada como parte importante de las élites locales o de los partidos políticos. En estos casos, hacemos referencia a una historia oficial, desde arriba, que revela la existencia una juventud política, vital y con expectativas de renovación y relevo generacional como en el caso de los estudiantes hasta mitad del siglo XX. Los otros jóvenes, los de abajo, no aparecen relacionados más que en historias de la delincuencia, los datos de los integrantes de los ejércitos en las contiendas o los desocupados. Esta aproximación general se apoya en la investigación previa y continua realizada por el autor del texto. ${ }^{1}$

\section{Rastros de la Juventud en la historia colombiana. El inestable siglo XIX.}

El siglo XIX en Colombia se puede dividir conforme a su organización política, la cual nos sirve como marco referencial para encontrar esas huellas de la juventud:

\begin{tabular}{|l|l|l|}
\hline Periodo & Denominación & Característica política \\
\hline $1739-1810$ & Virreinato de la Nueva Granada. & Colonia española \\
\hline $1811-1816$ & $\begin{array}{l}\text { Provincias Unidas de la Nueva } \\
\text { Granada }\end{array}$ & Centralismo - Federalismo \\
\hline $1816-1819$ & Virreinato de la Nueva Granada & Colonia española \\
\hline $1819-1830$ & Gran Colombia & $\begin{array}{l}\text { Unión de Venezuela, Quito y Nueva } \\
\text { granada }\end{array}$ \\
\hline $1831-1858$ & Estado de la Nueva Granada & Estado centralista \\
\hline $1858-1863$ & Confederación Granadina & Estado Federal \\
\hline $1863-1886$ & Estados Unidos de Colombia & Estado Federal \\
\hline $\begin{array}{l}1886 \\
\text { adelante en }\end{array}$ & República de Colombia. & República Centralista. \\
\hline
\end{tabular}

Cuadro No 1. Elaboración del Autor.

Así como cambió varias veces el nombre del país, también lo hicieron sus referentes geográficos y culturales. La manera como se vieron así mismas las poblaciones difiere de un momento a otro y esos marcos políticos y territoriales sirvieron para construir referentes de nación y de sus ciudadanos. El punto de

\footnotetext{
${ }^{1}$ La Investigación "Historia de los Jóvenes en Colombia: 1903-1991", fue realizada por el autor en el marco de su tesis doctoral en historia, terminada en 2012, en la Universidad Nacional de Colombia. A partir de allí, el autor ha publicado dos libros financiados por la Universidad Distrital Francisco José de Caldas: Historia, Jóvenes y Política (2014) y (Jóvenes, reclutas y Desertores 1819-1960 (2017).
} 


\section{Carlos Arturo Reina Rodríguez}

partida lo hallamos en el proceso de emancipación que tuvo como preludio a fines del siglo XVIIII, en las reuniones de varios grupos literarios y sociedades de jóvenes, hombres y algunas mujeres que se proclamaron como libres, en ciudades como Tunja y Bogotá. Unos eran peninsulares, otros criollos educados en Europa, y con acceso a la literatura europea no española, de tal manera que podían discutir, traducir y divulgar las ideas liberales y revolucionarias derivadas de las revoluciones francesa y estadounidense. Gracias a la imprenta, Antonio Nariño hizo la traducción de los Derechos Universales del Hombre en 1793, la cual inspiró a los jóvenes criollos, a la búsqueda de mejores condiciones políticas por parte de la metrópoli española. Estos son los jóvenes a los que el historiador Renán Silva denomina como "juventud noble del reino", precursores de la independencia nacional ${ }^{2}$.

Como adultos jóvenes, participaron en la declaración de independencia del 20 de Julio de 1810, así como en la primera guerra civil entre 1811 y 1814, conocida en la historia del país como "Patria Boba". La mayor parte de ellos murieron en esa contienda, motivo por el que algunos aciertan en denominar a aquellos mártires como pertenecientes a una "generación trágica". Los demás, fueron fusilados o condenados a las mazmorras al momento de la reconquista española en 1816: Camilo Torres, Francisco José de Caldas, Antonio Nariño, Francisco Antonio Zea, Policarpa Salavarrieta. Eran líderes inexpertos en el arte de la guerra tanto como en el de la política, pero tuvieron el ánimo y la vitalidad para enfrentar a los españoles y aprovechar las condiciones que se dieron mientras España era ocupada por los franceses. Los Ilustrados, formaron parte de ese caudal juvenil que pasó la mayor parte de su vida en la zozobra al ser declarados insurgentes y enemigos del gobierno español. No valieron las súplicas ni el reconocimiento de su gran valor como científicos y pensadores, pues durante la reconquista, algunos fueron fusilados por órdenes del Virrey Juan Sámano. La guerra se consumó el 7 de agosto de 1819 con el triunfo patriota en la batalla de Boyacá, de tal manera que el venezolano Simón Bolívar fue nombrado presidente mientras que su segundo al mando, Francisco de Paula Santander, asumió como vicepresidente a la edad de 27 años.

En esa batalla se destacó Pedro Pascasio Martínez ${ }^{3}$, quien capturó al comandante de las fuerzas españolas, el general Barreiro, que al verse acorralado, ofreció al joven una suma de dinero a cambio de su libertad. A pesar de ello, el soldado lo entregó a las fuerzas patriotas. Gracias a esto fue ascendido a Sargento. La presencia de menores en la guerra de independencia obedeció a que las fuerzas revolucionarias echaron mano de todos aquellos que pudieran empuñar las armas, incluyendo algunas mujeres y negros libertos.

Con la expulsión de los españoles, se dio paso a la Gran Colombia, que unió a Nueva Granada, Venezuela y Quito. Durante ese periodo (1819-1830), las tropas

\footnotetext{
2 Renán Silva. Los ilustrados de Nueva Granada 1760-1808. Genealogía de una comunidad de interpretación. Medellín: Fondo Editorial EAFIT Banco de la República 2002.

${ }^{3}$ Se estima que su edad estaba entre los 12 a los 14 años. Es conocido como el "niño - Soldado" y su nombre lo lleva un batallón del ejército colombiano.
} 


\section{Las juventudes en la historia Colombiana del siglo XIX y XX}

granadinas fueron integradas por jóvenes que buscaban una oportunidad para participar en las gestas libertadoras, y lucharon en los territorios del Perú y el Alto Perú.

A partir de 1830, apareció una nueva generación de jóvenes que creció en un país que abandonaba la guerra y se disponía a sanear sus finanzas para consolidar su unidad. A pesar de ello, el fantasma de la guerra apareció en 1839 cuando se dio inicio a la llamada "Guerra de los Supremos". En ella, los terratenientes de varias regiones del país se opusieron al gobierno central, cuando este quiso expropiar algunos conventos en el sur del país. Se presentaron sublevaciones en todo el territorio, hubo dimisión del presidente José Ignacio Márquez, e incluso tropas ecuatorianas ingresaron al país. La guerra terminó en 1842, sin embargo, una de sus características fue la de emplear como soldados a campesinos, jornaleros, esclavos y en general, todo hombre, joven y niños dispuesto a seguir al "Supremo", o líder regional. Los relatos señalan que participaron algunas mujeres en ambos bandos, para defender sus posesiones y a sus hijos. Esta guerra configuró lo que en adelante daría lugar a los partidos políticos Liberal y Conservador ${ }^{4}$.

A su alrededor se constituyeron las llamadas sociedades democráticas, que, al estilo de otras similares nacidas en países como Argentina, se fueron constituyendo por todo el país. Fueron, la Sociedad Filotémica, abiertamente conservadora y la Escuela Republicana, de corte liberal, las que contuvieron a las juventudes de la época. La mayoría de sus integrantes eran jóvenes procedentes de sectores aristocráticos, cuyos padres habían peleado en las guerras de independencia, y aunque los admiraban por tal gesta, también los consideraban como "viejos" para administrar los destinos del país. Afirma Cortés que "Todos ellos, en tiempos del presidente Mosquera bebieron de las fuentes de la Ilustración y ampliaron su horizonte académico al conocer los nuevos desarrollos del pensamiento en la Europa decimonónica, representados en las obras de autores como Lamartine, Blanc, Bentham, Fourier, Saint-Simon, Comte, Say, Bastiar, Proudhon, Constant, Cabet, y los novelistas Víctor Hugo y Eugène Sue, entre otros. Una juventud poseedora de una fuerte e inquieta rebeldía, acompañada de una gran curiosidad por conocer los nuevos rumbos del pensamiento" 5 .

Este proceso se había iniciado gracias a las reformas educativas impulsadas por Francisco de Paula Santander en la década de los años 20, cuando aparecieron las primeras escuelas oficiales y se inició el largo y lento proceso de alfabetización, que dividió a la educación en dos grandes niveles: Los que estudiaban en las escuelas y colegios rurales y los que ingresaban a los grandes colegios de la república, ubicados generalmente en ciudades como Bogotá. Así apareció una de esas formas a través de las cuales han sido más visibles los jóvenes: "el estudiante". Básicamente

\footnotetext{
${ }^{4}$ Estos son los partidos históricos en Colombia. Surgen a finales de la década de los 40 del siglo XIX.

${ }^{5}$ Luisa F. Cortes. Jóvenes y juventudes durante las reformas de medio siglo XIX: el caso de la Escuela Republicana. En: Luisa F. Cortés y Carlos A. Reina. Historia, Juventudes y Política: De la escuela republicana del siglo XIX a las élites y juventudes políticas de los gobiernos del siglo XX en Colombia. Bogotá. Universidad Distrital. 2014.
} 


\section{Carlos Arturo Reina Rodríguez}

se iba a la escuela porque eso daba prestigio, y porque el objetivo era llegar a los cuadros políticos del país.

Con la inestabilidad política, y la inauguración de la república federal en 1863, el país pasó a llamarse "Estados Unidos de Colombia". Hasta 1885, los liberales ocuparon el poder, en lo que también se conoce como el periodo del "Radicalismo"6. Entre muchas de sus políticas, iniciaron procesos contra la Iglesia a la cual expropiaron, expulsando además a los jesuitas del país. Importaron además, modelos de desarrollo y cambios educativos que llevaron a que en 1876 se desatara la llamada "Guerra de las Escuelas", posiblemente la única guerra en el mundo donde se enfrentaron dos facciones, la mayoría jóvenes estudiantes, por la implementación de un modelo pedagógico. Se trataba del modelo pestaloziano, que reemplazaba al de la pedagogía católica española. La guerra duró dos años.

La guerra inicio en 1876, y enfrentó al gobierno radical de Aquileo Parra contra el alzamiento en el Estado del Cauca, de tendencia conservadora. Las causas son múltiples e incluso algunos historiadores las ubican en políticas o decisiones generadas desde 1850. Durante dos años, se movilizó buena parte de los hombres en edad para la guerra, e incluso, se vincularon sectores de jóvenes de colegios prestigiosos, que hasta ese momento se habían mantenido al margen de las confrontaciones bélicas. Si bien a las Sociedades Democráticas de mitad del siglo XIX colombiano se habían integrado buen número de jóvenes, estos pertenecían a las élites políticas y por lo general no se vinculaban a los conflictos, rara vez tomaban las armas. Lo suyo era la palabra.

Caso aparte fue la vinculación de manera voluntaria de buena parte de los jóvenes pertenecientes a los sectores más acomodados, en la que, posiblemente haya sido su única participación como bloque de clase social enfrentados políticamente: unos liberales llamados Alcanfores, y otros conservadores, denominados Mochuelos. Los primeros fueron llamados de esa manera porque según las crónicas, -se evaporaban como el alcanfor, al calor de la batalla7. Esta afirmación nace de una descripción mucho más amplia que realizó el periódico El Alcanfor, después del final de la guerra en el año de 1877 y que en realidad da cuenta de la muerte de niños y jóvenes, su sacrificio y la gran pérdida para el país:

La Juventud que ocupaba los claustros de la Universidad y del Colegio del Rosario, viendo amenazada con aquella revolución su existencia moral, cerró los libros, voló a los parques en solicitud de armas, se organizó en Cuerpo, y se presentó en Palacio diciéndole al Presidente: Aquí estamos: jen donde es que se tiene necesidad de nuestro sacrifico!. El Presidente pudo haberse reído de aquella estudiantada y darles para sus dulces. Había entre ellos niños de diez años casi del tamaño de la bayoneta de su Remington. ¡Niños, pichones de héroes! ¡Seguramente aprendieron en el Catón el secreto de la inmortalidad! ...El presidente les dio cuartel y pocos días después pasó revista de comisario al

\footnotetext{
${ }^{6}$ Se conoce como Radicalismo por los cambios aperturistas y liberales llevados a cabo durante este periodo, la mayoría imitando las políticas inglesas y estadounidenses, pero con resultados poco favorables.

${ }^{7}$ Banco de la República. Alberto Urdaneta, vida y Obra. Bogotá. Biblioteca Luis Ángel Arango. 1992.
} 


\title{
Las juventudes en la historia Colombiana del siglo XIX y XX
}

Batallón -Libres de Colombiall. Un chusco de los muchos que hay entre los enemigos del gobierno, tuvo la chascada de decir, que al ver desfilar ese Batallón de niños, que se evaporaría como el alcanfor en el primer momento de peligro. $Y$ la chuscada produjo un efecto tal, que con el nombre alcanfor se conformaron hasta los mismos soldados niños que formaban el Batallón candidato para una evaporación próxima. Y el chusco no fue solamente chusco: fue Profeta; los niños se vaporaron, no ante el peligro, sino en él: Los cadáveres de más de sesenta de ellos, han quedado en los páramos de Guasca, en las llanuras de Garrapata, en los bosques de la Donjuna (sic) y en las cuevas sombrías de Manizales. Los niños enseñaron a muchos viejos cómo es que se saca provecho de la vida que apenas comenzaban a conocer: dándola en holocausto a la Patria cuando esta se ve amenazada por los hombres de las tradiciones, que viven prendidos de un pasado que no tiene ya derecho a la existencia!

Los segundos en contienda, recibieron el nombre de los Mochuelos, a propósito del nombre de la hacienda donde se reunieron al sur de Bogotá, aunque en realidad formaron parte del llamado Escuadrón Urdaneta. Enrique de Narváez describe algunos de esos enfrentamientos, describiendo a los dos bandos a partir de su gallardía y nobleza, que dista de aquella que tuvieron los reclutas comunes. Narváez recuerda el vínculo a ese escuadrón con un tinte de romanticismo y evoca de manera nostálgica algunos momentos de la vida cotidiana de las juventudes bogotanas al momento de vincularse a las guerrillas:

\begin{abstract}
De ese modo la juventud de Bogotá, esa ardorosa y noble juventud que no había tenido hasta entonces otro campo de disputa que la galantería y la gentileza en los salones de la sociedad; el del ingenio y espiritualidad en atrayentes círculos sociales; el de la destreza de las carreras de caballos, en las bulliciosas y alegres cuadrillas con que se festejaban en las plazas nuestras fiestas patrias, o bien el de la habilidad y resistencia en la esgrima, la gimnasia, el base ball y otros entretenimientos análogos, toda esa juventud, repito, participó y se contaminó del entusiasmo bélico que se había apoderado del país entero, y los que habían sido compañeros íntimos en esas lides se separaron, sin rencor ni odio, y más bien entre abrazos de sincero cariño para ir, los unos, a acrecentar las huestes del gobierno, formando aguerridos batallones, los otros, abandonando ciudad y hogares, para ir a distantes lugares y en ellos improvisar núcleos de defensa de la revolución ${ }^{9}$.
\end{abstract}

Durante el siglo XIX, el reclutamiento involucró a la población joven del país. Al parecer, la mayoría de jóvenes no querían ingresar a las filas de los ejércitos en contienda, sin embargo eran llevados en muchos casos, a la fuerza, no contaban con adiestramiento y eran sometidos a grandes esfuerzos, por lo que el número de desertores era muy alto ${ }^{10}$. Se dice que incluso algunos eran llevados amarrados al combate, mientras otros eran amenazados con perder a su familia o sus posesiones sino participaban en las guerras a las que eran convocados. Si existe una forma de

\footnotetext{
8 El Alcanfor. Bogotá. 1877. Octubre 6. Número 1. P.1.

${ }^{9}$ Enrique De Narváez. Los Mochuelos. Bogotá. Publicaciones del Ministerio de Educación Nacional.

Editorial Minerva. 1936, p. 30.

${ }^{10}$ Carlos Arturo Reina R. Jóvenes, reclutas y Desertores en Colombia. Siglos XIX y XX. Fondo editorial Universidad Distrital Francisco José de Caldas. 2017.
} 


\section{Carlos Arturo Reina Rodríguez}

ver a la juventud durante el siglo XIX en Colombia, esta puede ser la de su participación en cualquiera de las guerras que azotaron su territorio.

La edad fue un aspecto importante. Desde 1819 se aceptó una edad mínima para el ingreso al ejército nacional de 16 años, pero esta norma pocas veces se cumplió. Bastaba la firma de los padres para que niños menores de 10 años se incluyeran en las filas. Ellos prestaban servicio por cuatro años. El historiador Nelson Ramírez indica que, para el caso del Estado de Santander ubicado al oriente del país, el Código establecía que serían vinculados todos los varones mayores de 18 años y hasta los sesenta años. Comenta que existían dos fuerzas: una que era permanente y otra que era de reserva. Quedaban excluidos los médicos, los sacerdotes y pastores religiosos, los practicantes y sirvientes de los establecimientos públicos de beneficencia y caridad, lo mismo que aquellos que estaban impedidos física o mentalmente, así como los profesores y empleados públicos entre otros ${ }^{11}$. El gobierno central por lo general manejaba una lista para la incorporación de reclutas y para eliminar a quienes por cuenta de su fallecimiento o de alguna incapacidad no debía ser tenido en cuenta. Ante una eventualidad el reclutamiento forzado era generalizado a pesar de contar con el rechazo de la población.

En caso de que el reclutamiento operara en un hombre de una posición reconocida, por lo general, este era nombrado en algún cargo de oficialidad a pesar de carecer de formación militar. Los grados militares se dieron por los logros reconocidos en las confrontaciones militares, por órdenes de los altos oficiales, pero no por el ejercicio de una carrera militar. Apuntó Tomás Rueda Vargas, que - "De este año (1860) en adelante la cosecha de Generales ha venido en un aumento aterrador; cada guerra civil ha dado la suya, siendo de notarse que cada nueva cosecha es más numerosa y de peor calidad que la anterior"12

En algunos casos el ingreso a la vida militar se hacía gracias a que existía un legado familiar previo. Este es el caso del general Benjamín Herrera. Él estuvo en contacto desde un principio con la vida militar dado que su padre también lo había sido. En 1865, a los 15 años de edad, se presentó voluntariamente a la Guardia Colombiana, nombre con el que el radicalismo designó al ejército federal. Fue incorporado a la banda del regimiento y su vocación se expresó en boca de sus compañeros entre ellos Jorge Martínez Landínez quien señaló que:

Adolescente sentó plaza en la Guardia Colombiana, en cuyas filas fue muy pronto oficial de guerra, por su valor, espíritu militar y consagración al estudio del Código Militar, que entonces comprendía todos los reglamentos de las distintas armas; las Órdenes Generales que sabía de memoria; y los principios de Derecho de gentes, aprobados por las Convenciones Internaciones ${ }^{13}$.

\footnotetext{
11 Nelson Ramírez. Poder Civil y Ejército: Estado soberano de Santander 1857-1885. Tesis de Maestría en Historia. Bucaramanga. Universidad Industrial de Santander. 2010.

12 Rueda Vargas, Tomás. Escritos. Agosto 31 de 1909. Bogotá. Antares Limitada. Imprenta Fotograbado. 1963, p. 207.

${ }^{13}$ Gilberto Silva en: Revista Universidad Libre. 3ra época No 4. 1955 p, 81. Citado por Gustavo Humberto Rodríguez. Benjamín Herrera. En la Guerra y en La Paz. . Bogotá. Universidad Libre 1973.
} 


\section{Las juventudes en la historia Colombiana del siglo XIX y XX}

\section{Jóvenes y siglo $\mathrm{XX}$}

Durante el siglo XX se presentó una transformación demográfica que llevó a que la población joven aumentara en el país, producto de la disminución de la mortalidad generada por los distintos procesos de desarrollo, según explica Parra Sandoval ${ }^{14}$ Coincide además con los estudios que Carmen Elisa Flórez realizó en el año 2000 acerca de las transformaciones sociodemográficas en Colombia durante el siglo XX, que indican que en 1904 la población de menores de 15 años representaba el 40\%, y que esta aumentó a 45\% en 1964 y se redujo a 34.5\% en 1993. Agrega que este comportamiento de la población joven implicó una evolución contraria de la población entre 15 y 64 años cuya participación disminuyó hasta 1964, llegando a un 52\%, y de allí en adelante inició un aumento hasta el 61\% en 199315. En el periodo de estudio se observa entonces un ascenso de la población joven hasta los años 60 y un declive en adelante, que señala que el paso de altos a bajos niveles de fecundidad y mortalidad ha tenido un impacto demográfico directo sobre la estructura por edad de la población, la cual tiende a envejecerla.

El siglo XX, se inició con la contienda de la Guerra de los Mil Días (1899-1902). El enfrentamiento provocado por los partidos tradicionales, llevó a que miles de hombres, mujeres y niños fueran sacrificados en pos de las luchas partidistas. Luego de ello, en 1903, se estableció la Ley 39 que organizó las escuelas y se enfocó en preparar a los jóvenes para el desarrollo de actividades técnicas, como estrategia para evitar que se involucraran en futuras contiendas. Al mismo tiempo se organizó la profesión militar y el gobierno se dio a la tarea de abrir puestos de trabajo para la reconstrucción del país. La guerra civil provocó entre otras cosas la pérdida del departamento de Panamá, tras la intervención estadounidense, lo que creó una conciencia patriótica que no duró mucho.

Este siglo puede ser caracterizado por la presencia de los jóvenes en varios campos: Como estudiantes, fuerza juvenil dinamizadora de la vida social, y cultural del país. En 1908, aparecen los primeros movimientos estudiantiles. En 1909, se convocó al Primer Congreso Gran colombiano de estudiantes, que vinculó a estudiantes de los antiguos estados que la compusieron: Colombia, Ecuador y Venezuela. El evento se realizó en 1910, a propósito del primer centenario de la independencia y de él se desprendió un documento que planteaba la necesidad de unificar a todos los movimientos estudiantiles del continente. Se conocieron como la "Generación del centenario", criticada por los estudiantes de los años 20, quienes los consideraban como defensores del legado decimonónico. Estuvo integrada entre otros por Luis López de Mesa, Tomás Rueda Vargas, José Eustaquio Rivera, Calixto Torres, Rafael Escallón, Luis Augusto Cuervo, Antonio Álvarez Lleras, y los futuros pintores Ricardo Gómez Camuzano y Coriolano Leudo.

\footnotetext{
14 Rodrigo Parra S. La juventud colombiana. Bogotá. Plaza \& Janes. 1985, pp. 23-28.

15 Carmen E. Flórez y Regina Méndez. Las transformaciones sociodemográficas en Colombia durante el siglo XX. Bogotá. Banco de la República. 2000, p.6.
} 


\title{
Carlos Arturo Reina Rodríguez
}

Según Agustín Nieto Caballero, los "centenaristas" tuvieron una influencia francesa y se declararon como una generación de lectores, formados alrededor de la revista Cultura, la cual solo tuvo curiosamente solo 6 suscriptores $^{16}$. Lo que los unió fue

En principio la literatura, porque el eje del grupo fue la revista Cultura, que no se leía o no leíamos sino nosotros. La revista servía para sacar en limpio lo que escribíamos. Y con una botella de champaña alcanzó para todos. Los suscriptores eran 6. Pero ahí proyectamos prácticamente la vida: López de Mesa, sería filósofo, Rivas historiador, Gustavo Santos, crítico de arte, LENC (Luis Eduardo Nieto Caballero) periodista y yo educador. ${ }^{17}$

En 1910 se fundó la Escuela de Bellas Artes de Medellín. Allí se encontraron Ricardo Rendón, caricaturista, Francisco Cano, Félix Mejía Arango, escritor y caricaturista), Teodomiro Izasa, poeta, pintor y caricaturista, y Bernardo Martínez Toro, músico y dibujante. En 1913 fueron expulsados de la Escuela de Minas, Jorge Villa Carrasquilla, Escritor, León de Greiff, escritor y político, Jesús Restrepo Olarte, Poeta, y Gabriel Uribe Márquez, quien fue excomulgado ese mismo año. Todos se conocieron debido a un enfrentamiento entre estudiantes simpatizantes del partido liberal y el partido conservador, en la Plazuela de San Ignacio el 11 de Mayo de 1913 en la ciudad de Medellín. Los liberales estaban dirigidos por el joven León de Greiff y los Conservadores por el sacerdote español Cayetano Sarmiento y por José Mora Vásquez. Por más de tres horas, los estudiantes se enfrentaron a piedras, situación que terminó con la intervención de la policía. Todos tenían en común el hecho de haber sido expulsados de distintas instituciones educativas, y algunos de haber sido excomulgados. Declarados lectores de Nietzsche y Baudelaire, la constitución de su grupo implicó la creación del primer movimiento literario y artístico modernista de Colombia. De Greiff y Márquez eran colaboradores del periódico liberal y anticlerical La Fragua. Afirma Miguel Escobar que:

\begin{abstract}
Otra de las causas de origen del grupo es de orden "geográfico": a los estudiantes (o por lo menos muy desaplicados), en vacancia temporal o permanente, y precoces bohemios metidos en la picaresca local, coinciden en frecuentar los mismos lugares en busca de copas, tertulia, canciones, billares y muchachas... de alguna reputación ligera (tanto los lugares como las muchachas). La lista incluye, el Monserrate, "un centro anexo a la Universidad", el Vesubio, junto a la Escuela de Minas, donde ejercía la virtuosísima la "Guapa", el Jordán, aun vivisobrante; las sancocherías con músicos Guanteros y los primeros burdelitos de Lovaina ${ }^{18}$.
\end{abstract}

\footnotetext{
${ }^{16}$ El número de lectores expresa lo limitado del ejercicio de la escritura, pero también el de las llamadas "generaciones literarias" como en el caso de "Los Nuevos". Por lo tanto, tampoco fueron movimientos masivos.

${ }^{17}$ Agustín Nieto Caballero. “Centenarista Clásico” En: El Tiempo 5 febrero 1968 Bogotá, p. 24.

${ }^{18}$ Miguel Escobar Calle. Los Panidas de Medellín, Crónica sobre el grupo literario y su revista de 1915. Revista Credencial Historia. Bogotá. Credencial. Agosto 19 de 2004. Versión Digital.
} 


\title{
Las juventudes en la historia Colombiana del siglo XIX y XX
}

Con ellos, se fundó la revista "Panida" en 1915, en Medellín. El grupo de la revista se había formado un año antes y estuvo conformada en total por 13 jóvenes, límite propuesto por ellos mismos. Tenían entre 18 y 20 años. El 15 de Junio circuló el primer número de la revista. Las reacciones en Medellín fueron variadas. Unas de júbilo, otras de crítica y las últimas, por parte del órgano oficial de la curia llamado La Familia Cristiana en el que se vetó la revista y prohibieron a los jóvenes su lectura. Para Miguel Escobar "los Panidas, más que un grupo, fueron la primera y lúcida manifestación de una real e historia generación colombiana que luego se conocería con el nombre de Los Nuevos"19. La revista se terminó un año más tarde debido a que muchos de ellos se radicaron en Bogotá.

"Los Nuevos" aparecieron a principios de los años 20. Fueron un grupo de escritores jóvenes, no muy numeroso, que tuvo influencia en los círculos políticos y culturales de Bogotá y del país. Tres importantes personajes se vincularon a ellos: León de Greiff, Rafael Maya y Germán Pardo García. Coincidió la irrupción de estas publicaciones con el desarrollo del movimiento estudiantil, inspirado en el Movimiento de Córdoba Argentina de 1918 en Argentina. Lideró la formación de la primera asociación, el estudiante Germán Arciniegas, quien apoyado por el joven mexicano Carlos Pellicer, dio inicio a las actividades de un estudiantado organizado a partir de 1920. Escribió Arciniegas que

\begin{abstract}
La juventud o esos raros maestros que estudiando enseñan. La universidad, como organismo corriente, no estaba organizada para revisar conceptos: no podía hacer otra cosa que repetir verdades probadas por el tiempo, ideas antiguas, para llevar la antorcha del saber de las generaciones pasadas a las que apenas surgían. La universidad podía lavarse las manos y decir a los Nuevos: lo que viene queda por cuenta de ustedes. Para revisar conceptos era preciso establecer contactos con la vida: La universidad no los tenía 20
\end{abstract}

La asamblea de estudiantes trató que la vida estudiantil no se limitara a las clases y a los exámenes, y se proyectó como una "síntesis de la Patria" de quienes podrían regir el futuro del país. Aunque fueron criticados, lograron instaurar actividades extracurriculares y se propusieron abrir cursos gratuitos para los más pobres. Sin embargo lo más relevante estuvo en la organización del Carnaval Estudiantil, a modo de los que se realizaban en el mes de Octubre en otras capitales de América Latina. Durante una semana las calles de Bogotá y posteriormente de otras ciudades, se tornaban multicolor, con la algarabía, las comparsas, los desfiles y las actividades de los estudiantes, lo que trastornaba la cotidianidad y desencadenaba toda suerte de críticas, como la que señalaron las directivas del Colegio de San Bartolomé: "El señor Julio Jaramillo amenaza con la expulsión inmediata a aquel que llegara a disfrazarse. Aún más; los internos serán llevados a

\footnotetext{
19 Idem, Escobar.

${ }^{20}$ Germán Arciniegas. Los estudiantes de la mesa redonda. Bogotá. Edición 1982. p, 143.
} 


\section{Carlos Arturo Reina Rodríguez}

Zipaquirá, so pretexto de una excursión, para que no puedan ni siquiera ver el paseo de las máscaras"21

Junto a Los Nuevos aparecieron otros jóvenes llamados Los Leopardos, integrado por jóvenes conservadores que en realidad representaban a los sectores más moderados y moralistas del país. Unos y otros formarían parte de quienes lideraron al país a partir de 1930 cuando se inició la llamada República Liberal (1830-1845), luego de 44 años de gobiernos conservadores (1886-1930). Es importante anotar la presencia de mujeres como María Cano, quien impulsó una protesta por losd erechosd e los trabajadores y se opuso férreamente al reclutamiento de menores de edad en el país. A la edad de 26 años, María Cano fue distinguida como "flor del trabajo" y se hizo conocida por el periódico 888 (ocho horas de estudio, ocho horas de trabajo, ocho horas de descanso), órgano a través del cual planteó sus posturas políticas. Ella se convirtió en un referente de la clase obrera, motivo por el cual estuvo detenida en varias ocasiones, y hoy es ícono de la lucha por los derechos de las mujeres colombianas. Fue la voz femenina en un mundo donde apenas eran reconocidas como reinas estudiantiles, a pesar de que no estudiaban en las universidades ni eran estudiantes.

Ser estudiante reflejó admiración, esperanza y prevención. La literatura reflejó la cotidianidad del encanto de las "muchachadas" en distintos momentos. Hernando Téllez, escribió:

El grupo de muchachas venía a pie, por la ancha avenida. Traían los cabellos al
aire, las cabezas altas, erguidas, cándidamente desafiadoras. Bajo la tela de los
trajes, se percibía elástica y fina la línea de sus cuerpos, la eréctil poma de los
senos, el juego de los músculos. ...La juventud acababa de pasar por la calle, bajo
la preciosa y frágil envoltura carnal de la belleza femenina en perfecta sazón: Esas
muchachas simbolizaban fugazmente el triunfo de la vida sobre las asechanzas
del tiempo, del dolor, de la enfermedad, de la debilidad humana ${ }^{22}$.

La ropa, representada en el pantalón corto en el caso de los varones fue un elemento representativo cuando no se tenía la mayoría de edad que por entonces era a los 21 años. Cuenta Carlos Villar Borda en 1938 que

Durante un año utilicé un par de Sneakers, unos pantalones bombachos que llegaban hasta un poco más arriba del tobillo y que eran el último grito de la moda juvenil muy comunes en Inglaterra. Cuando nos echábamos largos", expresión coloquial para indicar el momento en que uno pasaba a usar pantalones largos, si las posaderas o los codos comenzaban a brillar demasiado, se mandaban voltear donde un sastre especializado en esta operación y así se les prolongaba la vida por lo menos un año. El único inconveniente era que el bolsillo del saco para el pañuelo venía a quedar a la derecha y todo el mundo lo notaba. También era frecuente teñirlos para darles una nueva existencia. Para los lutos, se teñían de negro. Desde luego que nunca estrenábamos traje porque siempre eran

\footnotetext{
${ }^{21}$ La fiesta estudiantil. El Tiempo. Bogotá. Septiembre 17 de 1924. p.3.

22 Hernando Téllez. Bagatelas. En: Sus mejores prosas. 1er festival del libro colombiano. Bogotá.

Biblioteca Básica de Cultura Colombiana. Bogotá. 1944, 16.
} 
"heredados" de parientes cercanos. Mi madre lograba descuentos o becas en los colegios donde íbamos a estudiar y lograba conseguir los libros de texto 23

Para muchos jóvenes, estudiar era un requisito que se fue haciendo necesario y con el tiempo una obligación, lo que no fue excusa para que se aprovecharan los llamados "tiempos mozos" en la conquista, los juegos, los bailes, y el café. Algunos lugares se fueron convirtiendo en las nuevas "salas" para conocer señoritas y los muchachos salidos del colegio o las universidades buscaban los lugares donde establecer vínculos con las muchachas de otras instituciones. Moreno narra que era el tranvía uno de los lugares donde se encontraban los enamorados, antes de la aparición de los modernos autobuses

Los novios deseaban en los paseos que se ocultara el sol y viniera la lluvia. Los enamorados se sentaban generalmente en los asientos traseros, y en de adelante, de doblar, se acomodaba la suegra o la tía de antiparras y mirada maliciosa. Cuando venía el chaparrón, la cortina de hule apenas lograba amparar a la pareja y la vieja soportaba, en medio de rabietas, la grosería de la lluvia. Y entonces el coche se convertía en una alcancía de escombros, porque dentro de aquella atmósfera artificialmente tibia que funcionaba bajo la cortina, cubriendo las extremidades de la pareja enamorada, las manos ansiosas jugaban una partida de fugas y encontrones, en un dulcísimo lenguaje táctil ${ }^{24}$

Los Cafés fueron los lugares obligados para los estudiantes y aunque eran públicos, un estudiante no podía sentarse en cualquier mesa sino solo cuando era invitado pues estas eran como oficinas para las reconocidas figuras de la sociedad bogotana. Estos fueron importantes hasta la década de los años 50, cuando el aumento de la población estudiantil fue dejando de lado aquellos lugares donde se escuchaba tango, se jugaba billar y se tomaba café.

En la década de los 30 aparecieron grupos de jóvenes que se interesaron por los eventos en España y en general en Europa. Se destacaron las Juventudes Católicas, las Juventudes Obreras, las juventudes liberales y las Juventudes conservadoras Alzatistas y Laureanistas, que seguían de cerca el proceso de la guerra civil española y la apoyaban. También aparecieron nuevos movimientos culturales, esta vez de carácter literario como las del grupo de Piedra y Cielo, que tomó el nombre de un libro de Juan Ramón Jiménez publicado en 1919, tuvo un impacto importante en la juventud colombiana pues marcó una transición hacia nuevas formas de entender la realidad a partir de la poesía así como de la prosa. La dinámica de las ciudades y los contrastes con los campos se vio reflejada en las obras de estos escritores que a su vez fueron los primeros en usar la radio como medio de difusión de la literatura, en particular en el caso de Gerardo Valencia. La influencia de los Piedra y Cielo, llegó hasta el punto que visitaron en 1944 el Liceo de Nacional de Varones en Zipaquirá donde estaba recién llegado de Barranquilla, Gabriel García

${ }^{23}$ Carlos Villar-Borda. La pasión del periodismo. Bogotá. Universidad Jorge Tadeo Lozano. 2004, 51.

${ }^{24}$ Idem Villar. p 36 


\section{Carlos Arturo Reina Rodríguez}

Márquez de 17 años, quien ya había publicado algunos escritos en la revista Juventud, en el Colegio San José de Barranquilla.

En 1937 apareció el movimiento artístico llamado Escuela Bachué, fundamental para analizar el cambio cultural de la perspectiva artística que esta vez miró hacia la representación de lo local. Integrada por jóvenes llegados de París, se opusieron a la reproducción del arte europeo y propugnaron por un arte propio. Allí se destacó la pintora Débora Arango con sus obras de desnudo, que fueron consideradas como irreverentes para aquella época.

Los periódicos y sobre todo las revistas funcionaron como puntos de encuentros para los escritores jóvenes en varios momentos de la historia colombiana del siglo XX. Como se indicó antes, en la década de los años 20, Luis López de Mesa había fundado con Agustín Nieto Caballero la revista Cultura. Germán Arciniegas publicó Universidad. León de Greiff Panida, Alberto Lleras Camargo Los Nuevos, y en los años 30 y 40, la Revista de Indias dirigida también por León de Greiff; la revista Pan, cuyo director era Enrique Uribe White en los años 40 y Mito en los años 50 creada por Jorge Gaitán Durán, además de Estrategia de Estanislao Zuleta y Mario Arrubla. En adelante se abrieron espacios para revistas académicas, donde su razón de ser, estuvo supeditada a la vinculación a centros académicos como las universidades. A través de la revista Mito, se articularon las generaciones de las décadas del 40 y el 50 con las de los años 60.

Fue allí donde Gonzalo Arango publicó por primera vez sus escritos. El principal exponente del nadaísmo cerró la última edición de la revista Mito en 1962, lo cual resultó tener un carácter simbólico frente a la transición generacional de los nuevos escritores. El movimiento nadaísta estuvo integrado por Gonzalo Arango, Amílcar Osorio (Amílcar U), Jaime Jaramillo Escobar (x-504), Eduardo Escobar J. Mario Arbeláez (J. Mario), Elmo Valencia, Humberto Navarro, Diego León Giraldo, Darío Lemus, Jaime Espinel, Alberto Escobar, Jorge Orlan y Moisés Melo entre otros. Se fundó a partir de la publicación del llamando Manifiesto Nadaísta, de Gonzalo Arango en el año de 1958. El folleto tenía 42 páginas y fue impreso en la tipografía "Amistad". Fueron una especie de intelectuales negativos. Gonzalo Arango esgrimía en su mano derecha una calavera. El primer título fue Sonata Metafísica para que Bailen los Muertos escrito por Arango. Su objetivo fue el de no dejar una fe intacta ni un ídolo en su sitio. Se tomaron La bastilla, un café en Medellín, a punta de recitales, Quemaron la urbanidad de Carreño, la María de Isaac, la Vorágine de José Eustaquio Rivera y los versos de Silva y Valencia, así como las fórmulas para alcanza la vida eterna del Padre Gaspar Astete.

Nacieron en Medellín, una ciudad que era el emblema de la defensa de los valores tradicionales y con elevado tradicionalismo, aferrada a una historia traída desde el siglo XIX a partir de la pujanza en tiempos de la colonización antioqueña. El pueblo de esta región se creyó bendecido por Dios, y por ende, el escudero de sus doctrinas y de la moral católica. Quizás por eso, Medellín fue en los 60 y principios de los 70, el campo de confrontación entre los jóvenes y las nuevas manifestaciones culturales, que resultaron subversivas a la luz de las poblaciones más adultas. 


\section{Las juventudes en la historia Colombiana del siglo XIX y XX}

Eran "marihuaneros adelantados a Woodstock. Eran poetas adelantados a su generación”, señalan José Hernández y Héctor Rincón ${ }^{25}$. Comenta Eduardo Escobar que "en nuestra infancia, la violencia ya estaba declinando pero era todavía el pan de cada día. El existencialismo apareció en la postguerra en Europa. El nadaísmo nació en nuestra post guerra. Claro, los nadaístas tratamos de no tener tema, ritmo, contenido... pero guardadas las proporciones el nadaísmo fue como un testimonio"26.

La segunda parte del siglo XX, había traído a los Nadaistas, pero también, con el ascenso de Gustavo Rojas Pinilla como dictador en 1953, había permitido formar un Movimiento estudiantil importante, que antes no tenía capacidad de respuesta. Luego de los hechos del 9 de abril de 1948, cuando fue asesinado Jorge Eliécer Gaitán, en el trágico "bogotazo", que dio origen a la Violencia (1949-1953), los estudiantes no habían logrado consolidar una posición política. Coincidió con el auge y apertura de nuevas universidades, la mayoría de carácter oficial, lo que aumentó el caudal de jóvenes en las aulas y la notoria presencia en las calles, ante el asesinato de algunos de ellos en 1954 y 1957. Fueron los estudiantes quienes impulsaron la salida del dictador en 1957, y posteriormente impulsaron las marchas de los años 60 hasta el año de 1971, año en el que se paralizaron las universidades públicas y privadas por cuenta de las manifestaciones estudiantiles que pedían participación en los espacios universitarios, en lo que se considera, el más amplio frente estudiantil de la historia colombiana.

De la misma manera y como en todo el continente, cientos de jóvenes inspirados en la revolución cubana, y en las ideas de izquierda, marcharon a los montes para dar origen a las guerrillas, buscando la toma del poder a semejanza del modelo cubano. Mientras tanto llegó el rock and roll, el hipismo, el feminismo y en general toda la parafernalia contracultural que tocó al mundo occidental. A pesar de que no fueron movimientos masivos y contaron con amplia resistencia entre los sectores tradicionales y conservadores del país, incidieron en las costumbres frente al vestuario y la vida cotidiana. Ancón (1971), el "Woodstock colombiano", se constituyó en el referente de aquel entonces ${ }^{27}$ a semejanza de otros en el continente.

Al mismo tiempo otros fenómenos atravesaron al país. La formación de carteles de tráfico de drogas ilícitas ocasionó que muchos jóvenes excluidos de las políticas oficiales de educación y trabajo, se vincularan como mano de obra barata de estos grupos. Medellín se fue convirtiendo en el eje de este negocio y con ello la aparición del sicariato que involucró a menores de edad llevó a que en 1984, un par de jóvenes asesinaran al Ministro de Justicia Rodrigo Lara Bonilla. Ese fue el momento en el que el país observo como se estaba asistiendo a la materialización de una generación de "No futuro", como lo retrató el cineasta Víctor Gaviria, eslogan

\footnotetext{
25 Héctor Rincón y Humberto Hernández. “De la Nada al Nadaísmo”. Lecturas Dominicales. El Tiempo. Bogotá 11 de sep. 1988, pp. 6-7.

${ }^{26}$ El ABC del Nadaísmo. En Lecturas Dominicales. El Tiempo. Bogotá 11 de Septiembre 1988, p7.

27 Reina Carlos (2012) Historia, Jóvenes y Memoria. Secretaría Distrital de Cultura. Bogotá.
} 


\section{Carlos Arturo Reina Rodríguez}

que coincidía con el del movimiento punk que se había afincado en Medellín y que desde luego estaba influenciado por el movimiento inglés.

A finales de la década de los 80 , la violencia tocó fondo. Tres candidatos presidenciales fueron asesinados, el Cartel de Medellín puso en jaque al Estado con atentados terroristas, secuestros y asesinatos selectivos. Por su parte las guerrillas arreciaban en los campos mientras se consolidaban las fuerzas paramilitares de autodefensas para enfrentarlas, ocasionando una debacle aún más grande. Cientos de jóvenes y niños fueron reclutados por los bandos en contienda. Miles murieron en los combates, en los atentados y en las refriegas donde también algunos estudiantes cayeron víctimas de la guerra. Paradójicamente, al mismo tiempo se levantaron voces juveniles contra la situación, desde escenarios radicales como los del mundo del underground en Medellín y Bogotá. Metaleros y Punkeros reaccionaron con canciones y letras de denuncia y crítica a la violencia de todo tipo, mientras que en los sectores menos radicales se impulsó desde la radio la expansión del "rock en tu idioma", el "rock en español", inspirado por las agrupaciones principalmente del sur del continente como Chile y Argentina. El "Concierto de Conciertos" realizado en septiembre de 1988, se convirtió en un hito generacional.

En medio de un ambiente enrarecido, en 1990, se inició el proceso de apertura económica que permitió la llegada de una avalancha de productos, bienes y servicios nunca antes vistos. Se consolidó el proceso de privatización de algunas empresas del Estado y se impulsó el consumo. Mientras tanto un grupo de estudiantes de universidades públicas y privadas, se movilizó para proponer una reforma constitucional, en las elecciones parlamentarias de ese año. El movimiento adquirió tal fuerza que se denominó Movimiento de la Séptima Papeleta. La iniciativa fue votada mayoritariamente y se citó a una Asamblea Nacional Constituyente que concluyó con el cambio de la constitución de 1886 y proclamó una nueva, donde se estableció el reconocimiento multicultural y el libre desarrollo de la personalidad, otorgando a los niños y los jóvenes, amplios derechos, que debían ser organizados jurídicamente. El diseño de una política para la infancia y la juventud fue sancionada años más tarde.

A pesar de que la academia dedicó más investigaciones acerca de los jóvenes, y que se constituyeron nuevas políticas públicas que otorgaron más espacios para los jóvenes, en la práctica muchos de ellos quedaron por fuera del sistema y el impacto comparado con la cantidad de estudios e investigaciones es todavía menor. El estudiantado disminuyó su participación en el último lustro del siglo, mientras expresiones como los eventos festivos vinculados a consumos culturales juveniles, fueron en aumento. El festival "Rock al "Parque", se convirtió en un punto referencial para el alcance de las políticas públicas aunque estas siguieron siendo limitadas. En términos del conflicto interno, el debate continuó relacionado con el vínculo de los menores de edad tanto en el Ejército Nacional como en los grupos al margen de la ley. Esto supuso la prohibición de reclutar a menores de edad en las fuerzas del Estado, y la solicitud a los demás grupos para que siguieran el mismo camino, cosa que quedó en ciernes. 


\section{En suma}

Como se advierte, la historia de la juventud colombiana se ha debatido en varias líneas de evidencia. Una, la que la vincula con los conflictos internos tanto en el siglo XIX como en el XX. Han sido carne de cañón de manera frecuente y aunque en el relato se especifica más sobre el siglo decimonónico, posiblemente el siglo que más sacrificios de jóvenes supuso fue el último.

Por otro lado, el estudiante ha sido un partícipe de esos momentos y coyunturas políticas que se han evidenciado aún más pasados la década de los 50 del siglo XX. Falta por reconstruir la historia del estudiantado del siglo XIX, no obstante, su presencia es escasa comparada con la del siglo siguiente, con lo cual se evidencia, no solo por la investigación sino por el número de trabajos al respecto, que es la forma más común para referirse a los jóvenes en la historia del país.

También, aunque han existido otras expresiones vinculadas al arte, la literatura, la música moderna y el deporte, estas son expresiones minoritarias o temporales, que, salvo los festivales masivos de finales del siglo XX, o los carnavales de los años 20 del mismo, apenas si son conocidas entre los jóvenes.

Así mismo se nota la ausencia de fuentes comparativas y ejercicios en ese sentido, para contrastar procesos en América Latina. En ese sentido conviene también la construcción de historias regionales que permitan tal ejercicio y puedan tejer puentes en común a partir de unidades que evidencien los lazos, similitudes, convergencias y diferencias entre unos y otros en el continente.

\begin{tabular}{|c|c|c|c|}
\hline PERÍODO & CARACTERÍSTICAS & MANIFESTACIÓN JUVENIL & REPRESENTANTES \\
\hline $\begin{array}{l}\text { Prehispánico } \quad \mathrm{y} \\
\text { Conquista }\end{array}$ & $\begin{array}{l}\text { Grupos indígenas diversos. } \\
\text { Conquistadores }\end{array}$ & $\begin{array}{l}\text { Forman parte de una clase, } \\
\text { vinculada a labores } \\
\text { económicas y militares. } \\
\text { Jóvenes bachilleres y } \\
\text { adelantados. }\end{array}$ & (Edades de los conquistadores) \\
\hline Colonia & $\begin{array}{l}\text { Dominio Español. Influencia de } \\
\text { las revoluciones Francesa y } \\
\text { Estadounidense. }\end{array}$ & $\begin{array}{l}\text { Jóvenes } \quad \text { ilustrados. } \\
\text { Sociedades Secretas. }\end{array}$ & $\begin{array}{l}\text { Antonio Nariño, Francisco José } \\
\text { de Caldas, } \\
\text { Francisco Antonio Zea. }\end{array}$ \\
\hline $\begin{array}{l}\text { República } \\
1810-1819\end{array}$ & $\begin{array}{l}\text { Grito de independencia. 1ra } \\
\text { guerra civil y reconquista } \\
\text { española. }\end{array}$ & Jóvenes Blancos criollos & $\begin{array}{l}\text { Francisco de Paula Santander, } \\
\text { José Miguel Fernández Madrid. }\end{array}$ \\
\hline $\begin{array}{l}\text { Gran Colombia } \\
1819-1830\end{array}$ & $\begin{array}{l}\text { Constitución de un Estado } \\
\text { formado por } \\
\text { Nueva Granada, Venezuela, } \\
\text { Quito y Panamá }\end{array}$ & Gobernantes Jóvenes. & \\
\hline $\begin{array}{l}\text { R. Nueva Granada } \\
1830-1858\end{array}$ & $\begin{array}{l}\text { Nueva Granada y Panamá } \\
\text { Gobierno centralista } \\
\text { republicano. } \\
\text { Varias guerras civiles. Modelo } \\
\text { Liberal. } \\
\begin{array}{l}\text { Formación de los partidos } \\
\text { políticos } \\
\text { Conservador. }\end{array} \\
\end{array}$ & $\begin{array}{l}\text { Jóvenes hijos } \text { de } r \text { los } \\
\text { precursores } \\
\text { independencia. de }\end{array}$ & $\begin{array}{l}\text { Sociedades Democráticas: } \\
\text { Escuela Filotémica y Sociedad } \\
\text { Republicana }\end{array}$ \\
\hline $\begin{array}{l}\text { Confederación } \\
\text { Granadina } \\
1858-1863 \\
\end{array}$ & Modelo Federalista. & & $\begin{array}{ll}\text { Juventudes } & \text { liberales } \\
\text { conservadoras } & \end{array}$ \\
\hline $\begin{array}{l}\text { Estados Unidos de } \\
\text { Colombia } \\
\text { 1863-1885 }\end{array}$ & $\begin{array}{l}\text { Modelo Federalista. } \\
\text { Proceso de Colonización } \\
\text { Antioqueña }\end{array}$ & Jóvenes de colegios de élite. & Guerra de las Escuelas \\
\hline
\end{tabular}


Carlos Arturo Reina Rodríguez

\begin{tabular}{|c|c|c|c|}
\hline $\begin{array}{l}\text { República de } \\
\text { Colombia } \\
\text { (Regeneración y } \\
\text { Hegemonía } \\
\text { Conservadora) } \\
\text { 1886-1930 } \\
\end{array}$ & $\begin{array}{l}\text { Regreso al modelo centralista. } \\
\text { Guerra de los Mil Días. } \\
\text { Separación de Panamá. }\end{array}$ & \begin{tabular}{l}
\multicolumn{3}{l}{ Generación del Centenario } \\
Pánidas. Generación de los \\
Nuevos. \\
Los Leopardos. 1er \\
Movimiento estudiantil
\end{tabular} & $\begin{array}{l}\text { León de Greiff, Germán } \\
\text { Arciniegas, Jorge Eliécer Gaitán, } \\
\text { María Cano, Antonio García } \\
\text { Nossa, Gonzalo Bravo Pérez. } \\
\text { Líder estudiantil asesinado. }\end{array}$ \\
\hline $\begin{array}{lr}\text { 1ra } & \text { República } \\
\text { Liberal } & \text { (1930- } \\
1945) & \end{array}$ & $\begin{array}{l}\text { Proceso de industrialización. } \\
\text { Guerra con el Perú. } 1932 .\end{array}$ & $\begin{array}{l}\text { Juventudes católicas, } \\
\text { juventudes conservadoras y } \\
\text { liberales. Escuela Bachue. }\end{array}$ & $\begin{array}{lrr}\text { Gilberto } & \text { Alzate } & \text { Avandaño, } \\
\text { Laureano } & \text { Gómez, } & \text { Debora } \\
\text { Arango, Gabriel García Márquez. }\end{array}$ \\
\hline $\begin{array}{l}\text { 2da República } \\
\text { Conservadora } \\
\text { 1945-1953 }\end{array}$ & $\begin{array}{l}\text { Violencia. Asesinato de Jorge } \\
\text { Eliecer Gaitán. Bogotazo. } \\
\text { Formación de las guerrillas de } \\
\text { los llanos y el Tolima. Batallón } \\
\text { Colombia. }\end{array}$ & $\begin{array}{l}\text { Jóvenes vinculados a } \\
\text { fuerzas militares y a } \\
\text { guerrillas campesinas. }\end{array}$ & $\begin{array}{l}\text { Teofilo Chispas, Pedro Antonio } \\
\text { Marín, Guadalupe Salcedo, } \\
\text { Soldados en Corea. }\end{array}$ \\
\hline $\begin{array}{l}\text { Dictadura Militar } \\
\text { 1953-1957 }\end{array}$ & $\begin{array}{l}\text { Control político militar por } \\
\text { parte del teniente General } \\
\text { Gustavo Rojas Pinilla. }\end{array}$ & Movimiento Estudiantil. & $\begin{array}{l}\text { Luchas estudiantiles y asesinato } \\
\text { de varios de ellos en } 1954 \text { y } \\
\text { 1957- }\end{array}$ \\
\hline $\begin{array}{l}\text { Frente Nacional } \\
(1958-1974)\end{array}$ & $\begin{array}{l}\text { Repartición del poder entre } \\
\text { liberales y conservadores, } \\
\text { durante } 16 \text { años. }\end{array}$ & $\begin{array}{l}\text { Movimientos Estudiantiles } \\
\text { Hippies, Rock and roll, } \\
\text { Feminismo. } \\
\text { Nadaismo. }\end{array}$ & $\begin{array}{l}\text { Jóvenes vinculados a las } \\
\text { guerrillas. Aumento de la } \\
\text { población estudiantil. } \\
\text { Festival de Ancón. (Woodstock } \\
\text { colombiano). Movimiento } \\
\text { Estudiantil de 1971.Luis Carlos } \\
\text { Galán. }\end{array}$ \\
\hline $1974-1990$ & $\begin{array}{l}\text { Desarrollo de los Carteles de } \\
\text { narcotráfico. } \\
\text { Estatuto de Seguridad. } \\
\text { Auge de las Guerrillas y } \\
\text { creación de las AUC. } \\
\text { Asesinato de Rodrigo Lara } \\
\text { Bonilla. 1984 }\end{array}$ & $\begin{array}{l}\text { Aparición de movimientos } \\
\text { Punk, Metal y Hip Hop. }\end{array}$ & $\begin{array}{l}\text { Construcción de la idea de la } \\
\text { generación del "No Futuro". } \\
\text { Película: Rodrigo D No futuro de } \\
\text { Víctor Gaviria. } \\
\text { Libro: Ausencia de futuro. } \\
\text { Rodrigo Parra Sandoval. }\end{array}$ \\
\hline 1991 & $\begin{array}{l}\text { Asamblea Constituyente. } \\
\text { Nueva Constitución de } 1991\end{array}$ & $\begin{array}{l}\text { Movimiento de la séptima } \\
\text { papeleta. } \\
\text { Reconocimiento del libre } \\
\text { desarrollo de la personalidad. } \\
\text { Aumento de las culturas } \\
\text { urbanas juveniles. }\end{array}$ & Sergio Fajardo \\
\hline
\end{tabular}

Cuadro No 2. Elaboración del autor.

\section{Bibliografía}

Agustín Nieto Caballero. Centenarista Clásico. En: El Tiempo 5 febrero 1968 Bogotá, p. 24.

Banco de la República. Alberto Urdaneta, vida y Obra. Bogotá. Biblioteca Luis Ángel Arango. 1992.

Carlos Villar-Borda. La pasión del periodismo. Bogotá. Universidad Jorge Tadeo Lozano. 2004, 51.

Carmen E. Flórez y Regina Méndez. Las transformaciones sociodemográficas en Colombia durante el siglo XX. Bogotá. Banco de la República. 2000, p.6.

El ABC del Nadaísmo. En Lecturas Dominicales. El Tiempo. Bogotá 11 de Septiembre 1988, p7.

El Alcanfor. Bogotá. 1877. Octubre 6. Número 1. P.1.

Enrique De Narváez. Los Mochuelos. Bogotá. Publicaciones del Ministerio de Educación Nacional. Editorial Minerva. 1936, p. 30.

Germán Arciniegas. Los estudiantes de la mesa redonda. Bogotá. Edición 1982. p, 143. 


\section{Las juventudes en la historia Colombiana del siglo XIX y XX}

Gilberto Silva en: Revista Universidad Libre. 3ra época No 4.1955 p, 81. Citado por Gustavo Humberto Rodríguez. Benjamín Herrera. En la Guerra y en La Paz. . Bogotá. Universidad Libre 1973.

Héctor Rincón y Humberto Hernández. “De la Nada al Nadaísmo”. Lecturas Dominicales. El Tiempo. Bogotá 11 de sep. 1988.

Hernando Téllez. Bagatelas. En: Sus mejores prosas. 1er festival del libro colombiano. Bogotá. Biblioteca Básica de Cultura Colombiana. Bogotá. 1944.

La fiesta estudiantil. El Tiempo. Bogotá. Septiembre 17 de 1924.

Luisa F. Cortes. Jóvenes y juventudes durante las reformas de medio siglo XIX: el caso de la Escuela Republicana. En: Luisa F. Cortés y Carlos A. Reina. Historia, Juventudes y Política: De la escuela republicana del siglo XIX a las élites y juventudes políticas de los gobiernos del siglo XX en Colombia. Bogotá. Universidad Distrital. 2014.

Miguel Escobar Calle. Los Panidas de Medellín, Crónica sobre el grupo literario y su revista de 1915. Revista Credencial Historia. Bogotá. Credencial. Agosto 19 de 2004. Versión Digital.

Nelson Ramírez. Poder Civil y Ejército: Estado soberano de Santander 1857-1885. Tesis de Maestría en Historia. Bucaramanga. Universidad Industrial de Santander. 2010.

Carlos Arturo Reina. Historia, Jóvenes y Memoria. Secretaría Distrital de Cultura. Bogotá. 2012.

Carlos Arturo Reina. Jóvenes, Reclutas y Desertores. Fondo editorial Universidad Distrital Francisco José de Caldas. Bogotá. 2017

Renán Silva. Los ilustrados de Nueva Granada 1760- 1808. Genealogía de una comunidad de interpretación. Medellín: Fondo Editorial EAFIT Banco de la República 2002.

Rodrigo Parra S. La juventud colombiana. Bogotá Editorial Plaza \& Janes. 1985

Tomás Rueda Vargas. Escritos. Agosto 31 de 1909. Bogotá. Antares Limitada. Imprenta Fotograbado. 1963.

Recibido: 01/10/2017

Evaluado: $13 / 12 / 2017$

Versión Final: 10/01/2018 